\title{
HARDY INEQUALITIES IN STRIPS ON RULED SURFACES
}

\author{
DAVID KREJČIŘÍK
}

Received 17 August 2005; Accepted 8 November 2005

We consider the Dirichlet Laplacian in infinite two-dimensional strips defined as uniform tubular neighbourhoods of curves on ruled surfaces. We show that the negative Gauss curvature of the ambient surface gives rise to a Hardy inequality and we use this to prove certain stability of spectrum in the case of asymptotically straight strips about mildly perturbed geodesics.

Copyright (c) 2006 David Krejčiřík. This is an open access article distributed under the Creative Commons Attribution License, which permits unrestricted use, distribution, and reproduction in any medium, provided the original work is properly cited.

\section{Introduction}

Problems linking the geometry of two-dimensional manifolds and the spectrum of associated Laplacians have been considered for more than a century. While classical motivations come from theories of elasticity and electromagnetism, the same rather simple models can be also remarkably successful in describing even rather complicated phenomena in quantum heterostructures. Here, an enormous amount of recent research has been undertaken on both the theoretical and experimental aspects of binding in curved striplike waveguide systems.

More specifically, as a result of theoretical studies, it is well known now that the Dirichlet Laplacian in an infinite planar strip of uniform width always possesses eigenvalues below its essential spectrum whenever the strip is curved and asymptotically straight. We refer to $[13,15]$ for initial proofs and to $[8,19,21]$ for reviews with many references on the topic. The existence of the curvature-induced bound states is interesting from several respects. First of all, one deals with a purely quantum effect of geometrical origin, with negative consequences for the electronic transport in nanostructures. From the mathematical point of view, the strips represent a class of noncompact noncomplete manifolds for which the spectral results of this type are nontrivial, too.

Hindawi Publishing Corporation Journal of Inequalities and Applications Volume 2006, Article ID 46409, Pages 1-10 DOI 10.1155/JIA/2006/46409 
At the same time, a couple of results showing that the attractive interaction due to bending can be eliminated by appropriate additional perturbations have been established quite recently. Dittrich and Kř́ž [7] demonstrated that the discrete spectrum of the Laplacian in any asymptotically straight planar strip is empty provided the curvature of the boundary curves does not change sign and the Dirichlet condition on the locally shorter boundary is replaced by the Neumann one. A different proof of this result and an extension to Robin boundary conditions were performed in [14]. Ekholm and Kovař́ík [10] obtained the same conclusion for the purely Dirichlet Laplacian in a mildly curved strip by introducing a local magnetic field perpendicular to the strip. The purpose of the present paper is to show that the same types of repulsive interaction can be created if the ambient space of the strip is a negatively curved manifold instead of the Euclidean plane.

A spectral analysis of the Dirichlet Laplacian in infinite strips embedded in curved two-dimensional manifolds was performed for the first time by the present author in [18]. He derived a sufficient condition which guarantees the existence of discrete eigenvalues in asymptotically straight strips; in particular, the bound states exist in strips on positively curved surfaces and in curved strips on flat surfaces. He also performed heuristic considerations suggesting that the discrete spectrum might be empty for certain strips on negatively curved surfaces. Similar conjectures were also made previously for strips on ruled surfaces in [5]. However, a rigorous treatment of the problem remained open.

In the present paper, we derive several Hardy inequalities for mildly curved strips on ruled surfaces, which proves the conjecture for this class of strips. A ruled surface is generated by straight lines translating along a curve in the Euclidean space; hence its Gauss curvature is always nonpositive. The reason why we restrict to ruled surfaces in this paper is due to the fact that the Jacobi equation determining the metric in geodesic coordinates is explicitly solvable, so that rather simple formulae are available. Nevertheless, it should be possible to extend the present ideas to other classes of nonpositively curved surfaces for which more precise information about geodesics are available.

Hardy inequalities represent a powerful technical tool in more advanced theoretical studies of elliptic operators. We refer to the book [22] for an exhaustive study and generalizations of the original inequality due to Hardy. Interesting Hardy inequalities on noncompact Riemannian manifolds were established in [2]. In the quantum-waveguide context, various types of Hardy inequality were derived in $[1,10,11]$ in order to prove certain stability of spectrum of the Laplacian in tubular domains.

Here the last reference is the closest to the issue of the present paper. Indeed, the authors of [11] considered a three-dimensional tube constructed by translating a noncircular two-dimensional cross-section along an infinite curve and obtained that the twisting due to an appropriate construction eliminates the curvature-induced discrete spectrum in the regime of mild curvature. Formally, the strips of the present paper can be viewed as a singular case of [11] when the cross-section is replaced by a segment and the effect of twisting is hidden in the curvature of the ambient space. While [11] and the present paper exhibit these similarity features, and also the technical handling of the problems is similar, they differ in some respects. On the one hand, the present situation is simpler, since it happens that the negative curvature of the ambient space gives rise to an explicit repulsive potential (cf. (3.6)) which leads to a Hardy inequality in a more direct way than 
in [11]. On the other hand, we do not perform the unitary transformation of [11] in order to replace the Laplacian on the Hilbert space of a curved strip by a Schrödingertype operator on a "straighten" Hilbert space, but we work directly with "curved" Hilbert spaces. This technically more complicated approach has an advantage that we need to impose no conditions whatsoever on the derivatives of curvatures.

Although we are not aware of a direct physical interpretation of the Laplacian in infinite strips if the ambient space has a nontrivial curvature, there exists an indirect motivation coming from the theory of quantum layers studied in $[3,9,20]$. In these references, the Dirichlet Laplacian in tubular neighbourhoods of a surface in the Euclidean space is used for the quantum Hamiltonian (cf. [12] for a similar model). Taking our strip as the reference surface, the layer model of course differs from the present one, but a detailed study of the latter is important to understand certain spectral properties of the former. Similar layer problems are also considered in other areas of physics away from quantum theories, (cf. [16]). Finally, the present problem is a mathematically interesting one in the context of spectral geometry.

The organization of the paper is as follows. The ambient ruled surface, the strip, and the corresponding Dirichlet Laplacian are properly defined in the preliminaries in Section 2. In Section 3, we consider the special situation of the strip being straight in a generalized sense. If the Gauss curvature of such a strip does not vanish identically and the strip is thin enough, we derive a central Hardy inequality of the present paper, (cf. Theorem 3.1). In fact, the latter is established by means of a "local" Hardy inequality, (cf. (3.7)), which might be also interesting for applications. In Section 4, we apply Theorem 3.1 to mildly curved strips and prove certain stability of spectrum, (cf. Theorem 4.1). As an intermediate result, we obtain a general Hardy inequality for mildly curved strips on ruled surfaces (cf. (4.7)).

\section{Preliminaries}

Given two bounded continuous functions $\kappa$ and $\tau$ defined on $\mathbb{R}$ with $\kappa$ being positive, let $\Gamma: \mathbb{R} \rightarrow \mathbb{R}^{3}$ be the unit-speed curve whose curvature and torsion are $\kappa$ and $\tau$, respectively. $\Gamma$ is determined uniquely up to congruent transformations and possesses a distinguished $C^{1}$-smooth Frenet frame $\{\dot{\Gamma}, N, B\}$ consisting of tangent, normal, and binormal vector fields, respectively (cf. [17, Chapter 1]). It is also convenient to include the case of $\kappa$ and $\tau$ being equal to zero identically, which corresponds to $\Gamma$ being a straight line with a constant Frenet frame.

Given a bounded $C^{1}$-smooth function $\theta$ defined on $\mathbb{R}$, let us introduce the mapping $\mathscr{L}: \mathbb{R}^{2} \rightarrow \mathbb{R}^{3}$ via

$$
\mathscr{L}(s, t):=\Gamma(s)+t[N(s) \cos \theta(s)-B(s) \sin \theta(s)]
$$

$\mathscr{L}$ represents a ruled surface (cf. [17, Definition 3.7.4]) provided it is an immersion. The latter is ensured by requiring that the metric tensor $G \equiv\left(G_{i j}\right)$ induced by $\mathscr{L}$, that is,

$$
G_{i j}:=\left(\partial_{i} \mathscr{L}\right) \cdot\left(\partial_{j} \mathscr{L}\right), \quad i, j \in\{1,2\}
$$




\section{Hardy inequalities in strips on ruled surfaces}

where the dot denotes the scalar product in $\mathbb{R}^{3}$, to be positive definite. Employing the Serret-Frenet formulae (cf. [17, Section 1.3]), we find

$$
G=\left(\begin{array}{cc}
h^{2} & 0 \\
0 & 1
\end{array}\right), \quad h(s, t):=\sqrt{[1-t \kappa(s) \cos \theta(s)]^{2}+t^{2}[\tau(s)-\dot{\theta}(s)]^{2}} .
$$

Hence, it is enough to assume that $t$ is sufficiently small so that the first term in the square root defining $h$ never vanishes.

More restrictively, given a positive number $a$, we always assume that

$$
a\|\kappa \cos \theta\|_{\infty}<1
$$

so that also $h^{-1}$ is bounded, and define a ruled strip of width $2 a$ to be the Riemannian manifold

$$
\Omega:=(\mathbb{R} \times(-a, a), G) .
$$

That is, $\Omega$ is a noncompact and noncomplete surface which is fully characterized by the functions $\kappa, \tau, \theta$ and the number $a$. It is easy to verify that the Gauss curvature $K$ of $\Omega$ is nonpositive, namely,

$$
K=-(\tau-\dot{\theta})^{2} h^{-4}
$$

Moreover, if the mapping $\mathscr{L}$ is injective, then the image $\mathscr{L}(\mathbb{R} \times(-a, a))$ has indeed the geometrical meaning of a non-self-intersecting strip and $\Omega$ represents its parameterization in geodesic coordinates.

Remark 2.1. In (2.3), let us write $k$ instead of $\kappa \cos \theta$ and $\sigma$ instead of $\tau-\dot{\theta}$, and assume that $k$ and $\sigma$ are given bounded continuous functions on $\mathbb{R}$. Then, abandoning the geometrical interpretation in terms of ruled surfaces based on $\Gamma$, (2.5) can be considered as an abstract Riemannian manifold, with $a\|k\|_{\infty}<1$ being the only restriction. The spectral results of this paper extend automatically to this more general situation by applying the above identification.

Our object of interest is the Dirichlet Laplacian in $\Omega$, that is, the unique selfadjoint operator $-\Delta_{D}^{\Omega}$ associated with the closure of the quadratic form $Q$ defined in the Hilbert space

$$
\mathscr{H}:=L^{2}(\Omega) \equiv L^{2}(\mathbb{R} \times(-a, a), h(s, t) d s d t)
$$

by the prescription

$$
Q[\psi]:=\left(\partial_{i} \psi, G^{i j} \partial_{j} \psi\right)_{\mathscr{H}}, \quad \psi \in D(Q):=C_{0}^{\infty}(\mathbb{R} \times(-a, a)),
$$

where $\left(G^{i j}\right):=G^{-1}$ and the summation is assumed over the indices $i, j \in\{1,2\}$. Given $\psi \in D(Q)$, we have

$$
Q[\psi]=\left\|h^{-1} \partial_{1} \psi\right\|_{\mathscr{H}}^{2}+\left\|\partial_{2} \psi\right\|_{\mathscr{H}}^{2}
$$


Under the stated assumptions, it is clear that the form domain of $-\Delta_{D}^{\Omega}$ is just the Sobolev space $W_{0}^{1,2}(\mathbb{R} \times(-a, a))$. If $\mathscr{L}$ is injective, then $-\Delta_{D}^{\Omega}$ is nothing else than the Dirichlet Laplacian defined in the open subset $\mathscr{L}(\mathbb{R} \times(-a, a))$ of the ruled surface (2.1) and expressed in the "coordinates" $(s, t)$.

\section{Geodesic strips}

The ruled strip $\Omega$ is called a geodesic strip and is denoted by $\Omega_{0}$ if the reference curve $\Gamma$ is a geodesic on $\mathscr{L}$. Since $\kappa \cos \theta$ is the geodesic curvature of $\Gamma$ (when the latter is considered as a curve on $\mathscr{L}$ ), it is clear that $\Omega$ is a geodesic strip provided that $\Gamma$ is either a straight line (i.e., geodesic in $\mathbb{R}^{3}$ ) or the straight lines $t \mapsto \mathscr{L}(s, t)-\Gamma(s)$ generating the ruled surface (2.1) are tangential to the binormal vector field for each fixed $s$. The metric (2.3) corresponding to $\Omega_{0}$ acquires the form

$$
G_{0}:=\left(\begin{array}{cc}
h_{0}^{2} & 0 \\
0 & 1
\end{array}\right), \quad h_{0}(s, t):=\sqrt{1+t^{2}[\tau(s)-\dot{\theta}(s)]^{2}}
$$

and we denote by $\mathscr{H}_{0}, Q_{0}$, and $-\Delta_{D}^{\Omega_{0}}$, respectively, the corresponding Hilbert space defined in analogy to (2.7), the corresponding quadratic form defined in analogy to (2.8), and the associated Dirichlet Laplacian in $\Omega_{0}$.

If $\tau-\dot{\theta}$ is equal to zero identically, that is, $\Omega_{0}$ is a flat surface due to (2.6), it is easy to see that the spectrum of $-\Delta_{D}^{\Omega_{0}}$ coincides with the interval $\left[E_{1}, \infty\right)$, where

$$
E_{1}:=\frac{\pi^{2}}{(2 a)^{2}}
$$

is the lowest eigenvalue of the Dirichlet Laplacian in $(-a, a)$. In this section, we prove that the presence of a Gauss curvature leads to a Hardy inequality for the difference $-\Delta_{D}^{\Omega_{0}}-E_{1}$, which has important consequences for the stability of spectrum.

THEOREM 3.1. Given a positive number $a$ and bounded continuous functions $\tau$ and $\dot{\theta}$, let $\Omega_{0}$ be the Riemannian manifold $\left(\mathbb{R} \times(-a, a), G_{0}\right)$ with the metric given by (3.1). Assume that $\tau-\dot{\theta}$ is not identically zero and that $a\|\tau-\dot{\theta}\|_{\infty}<\sqrt{2}$. Then, for all $\psi \in W_{0}^{1,2}(\mathbb{R} \times(-a, a))$ and any $s_{0}$ such that $(\tau-\dot{\theta})\left(s_{0}\right) \neq 0$,

$$
Q_{0}[\psi]-E_{1}\|\psi\|_{\mathscr{H}_{0}}^{2} \geq c\left\|\rho^{-1} \psi\right\|_{\mathscr{H}_{0}}^{2} \quad \text { with } \rho(s, t):=\sqrt{1+\left(s-s_{0}\right)^{2}} .
$$

Here $c$ is a positive constant which depends on $s_{0}, a$, and $\tau-\dot{\theta}$.

It is possible to find an explicit lower bound for the constant $c$; we give an estimate in (3.15) below.

Theorem 3.1 implies that the presence of a Gauss curvature represents a repulsive interaction in the sense that there is no spectrum below $E_{1}$ for all small potential-type perturbations having $O\left(s^{-2}\right)$ decay at infinity. Moreover, in Section 4, we show that this is also the case for appropriate perturbations of the metric (3.1). 
In order to prove Theorem 3.1, we introduce the function $\lambda: \mathbb{R} \rightarrow \mathbb{R}$ by

$$
\lambda(s):=\inf _{\varphi \in C_{0}^{\infty}((-a, a)) \backslash\{0\}} \frac{\int_{-a}^{a}|\dot{\varphi}(t)|^{2} h_{0}(s, t) d t}{\int_{-a}^{a}|\varphi(t)|^{2} h_{0}(s, t) d t}-E_{1}
$$

and keep the same notation for the function $\lambda \otimes 1$ on $\mathbb{R} \times(-a, a)$. We have the following lemma.

Lemma 3.2. Under the hypotheses of Theorem 3.1, $\lambda$ is a continuous nonnegative function which is not identically equal to zero.

Proof. For any fix $s \in \mathbb{R}$, we make the change of test function $\phi:=\sqrt{h_{0}(s, \cdot)} \varphi$, integrate by parts, and arrive at

$$
\lambda(s)=\inf _{\phi \in C_{0}^{\infty}((-a, a)) \backslash\{0\}} \frac{\int_{-a}^{a}\left(|\dot{\phi}(t)|^{2}-E_{1}|\phi(t)|^{2}+V(s, t)|\phi(t)|^{2}\right) d t}{\int_{-a}^{a}|\phi(t)|^{2} d t}
$$

with

$$
V(s, t):=\frac{[\tau(s)-\dot{\theta}(s)]^{2}\left(2-t^{2}[\tau(s)-\dot{\theta}(s)]^{2}\right)}{4 h_{0}(s, t)^{4}} .
$$

Under the hypotheses of Theorem 3.1, the function $V$ is clearly continuous, nonnegative, and not identically zero. These facts together with the Poincaré inequality $\int_{-a}^{a}|\dot{\phi}|^{2} \geq$ $E_{1} \int_{-a}^{a}|\phi|^{2}$ valid for any $\phi \in C_{0}^{\infty}((-a, a))$ yield the claims of the lemma.

Assuming that the conclusion of Lemma 3.2 holds and using the definition (3.4), we get the estimate

$$
Q_{0}[\psi]-E_{1}\|\psi\|_{\mathscr{H}_{0}}^{2} \geq\left\|h_{0}^{-1} \partial_{1} \psi\right\|_{\mathscr{H}_{0}}^{2}+\left\|\lambda^{1 / 2} \psi\right\|_{\mathscr{H}_{0}}^{2}
$$

valid for any $\psi \in C_{0}^{\infty}(\mathbb{R} \times(-a, a))$. Neglecting the first term on the right-hand side of (3.7), the inequality is already a Hardy inequality. However, for applications, it is more convenient to replace the Hardy weight $\lambda$ in (3.7) by the positive function $c \rho^{-2}$ of Theorem 3.1. This is possible by employing the contribution of the first term based on the following lemma.

Lemma 3.3. For any $\psi \in C_{0}^{\infty}(\mathbb{R} \times(-a, a))$,

$$
\left(1+a^{2}\|\tau-\dot{\theta}\|_{\infty}^{2}\right)^{-1 / 2}\left\|\rho^{-1} \psi\right\|_{\mathscr{H}_{0}}^{2} \leq 16\left(1+a^{2}\|\tau-\dot{\theta}\|_{\infty}^{2}\right)^{1 / 2}\left\|h_{0}^{-1} \partial_{1} \psi\right\|_{\mathscr{H}_{0}}^{2}+\left(2+\frac{64}{|I|^{2}}\right)\left\|\chi_{I} \psi\right\|_{\mathscr{H}_{0}}^{2}
$$

where $I$ is any bounded subinterval of $\mathbb{R}, \chi_{I}$ denotes the characteristic function of the set $I \times(-a, a)$, and $\rho$ is the function of Theorem 3.1 with $s_{0}$ being the centre of $I$. 
Proof. The lemma is based on the following version of the one-dimensional Hardy inequality:

$$
\int_{\mathbb{R}} \frac{|u(x)|^{2}}{x^{2}} d x \leq 4 \int_{\mathbb{R}}|\dot{u}(x)|^{2} d x
$$

valid for all $u \in W^{1,2}(\mathbb{R})$ with $u(0)=0$. Put $b:=|I| / 2$. We define the function $f: \mathbb{R} \rightarrow \mathbb{R}$ by

$$
f(s):= \begin{cases}1 & \text { for }\left|s-s_{0}\right| \geq b \\ \frac{\left|s-s_{0}\right|}{b} & \text { for }\left|s-s_{0}\right|<b\end{cases}
$$

and keep the same notation for the function $f \otimes 1$ on $\mathbb{R} \times(-a, a)$. For any $\psi \in C_{0}^{\infty}(\mathbb{R} \times$ $(-a, a))$, let us write $\psi=f \psi+(1-f) \psi$. Applying (3.9) to the function $s \mapsto(f \psi)(s, t)$ with $t$ fixed, we arrive at

$$
\begin{aligned}
\int \frac{|\psi|^{2}}{\rho^{2}} & \leq 2 \int \frac{|f \psi|^{2}}{\rho^{2}-1}+2 \int \chi_{I}|(1-f) \psi|^{2} \\
& \leq 16 \int\left|\partial_{1} f\right|^{2}|\psi|^{2}+16 \int|f|^{2}\left|\partial_{1} \psi\right|^{2}+2 \int \chi_{I}|(1-f) \psi|^{2} \\
& \leq 16 \int\left|\partial_{1} \psi\right|^{2}+\left(2+\frac{16}{b^{2}}\right) \int \chi_{I}|\psi|^{2}
\end{aligned}
$$

where the integration sign indicates the integration over $\mathbb{R} \times(-a, a)$. Recalling the definition of $\mathscr{H}_{0}$ and using the estimates

$$
1 \leq h_{0}^{2} \leq 1+a^{2}\|\tau-\dot{\theta}\|_{\infty}^{2}
$$

the lemma follows at once.

Now we are in a position to prove Theorem 3.1.

Proof of Theorem 3.1. It suffices to prove the theorem for functions $\psi$ from the dense subspace $C_{0}^{\infty}(\mathbb{R} \times(-a, a))$. Assume the hypotheses of Theorem 3.1 so that the conclusion of Lemma 3.2 holds. Let $I$ be any closed interval on which $\lambda$ is positive. Writing

$$
\left\|\lambda^{1 / 2} \psi\right\|_{\mathscr{H}_{0}}^{2}=\epsilon\left\|\lambda^{1 / 2} \psi\right\|_{\mathscr{H}_{0}}^{2}+(1-\epsilon)\left\|\lambda^{1 / 2} \psi\right\|_{\mathscr{H}_{0}}^{2} \quad \text { with } \epsilon \in(0,1]
$$

neglecting the second term of this decomposition, estimating the first one by an integral over $I \times(-a, a)$, and applying Lemma 3.3, the inequality (3.7) yields

$$
\begin{aligned}
Q_{0}[\psi]- & E_{1}\|\psi\|_{\mathscr{H}_{0}}^{2} \\
\geq & {\left[1-16 \epsilon \min _{I} \lambda\left(2+\frac{64}{|I|^{2}}\right)^{-1}\left(1+a^{2}\|\tau-\dot{\theta}\|_{\infty}^{2}\right)^{1 / 2}\right]\left\|h_{0}^{-1} \partial_{1} \psi\right\|_{\mathscr{H}_{0}}^{2} } \\
& +\epsilon \min _{I} \lambda\left(2+\frac{64}{|I|^{2}}\right)^{-1}\left(1+a^{2}\|\tau-\dot{\theta}\|_{\infty}^{2}\right)^{-1 / 2}\left\|\rho^{-1} \psi\right\|_{\mathscr{H}_{0}}^{2} .
\end{aligned}
$$


Choosing $\epsilon$ as the minimum between 1 and the value such that the first term on the right-hand side of the last estimate vanishes, we get the claim of Theorem 3.1 with

$$
c \geq \min \left\{\frac{\min _{I} \lambda}{\left(2+64 /|I|^{2}\right)\left(1+a^{2}\|\tau-\dot{\theta}\|_{\infty}^{2}\right)^{1 / 2}}, \frac{1}{16\left(1+a^{2}\|\tau-\dot{\theta}\|_{\infty}^{2}\right)}\right\} .
$$

\section{Mildly curved strips}

Recall that the spectrum of $-\Delta_{D}^{\Omega_{0}}$ coincides with the interval $\left[E_{1}, \infty\right)$ provided that the Gauss curvature (2.6) vanishes everywhere in the geodesic strip $\Omega_{0}$. On the other hand, it was proved in [18] that $-\Delta_{D}^{\Omega}$ always possesses a spectrum below $E_{1}$ provided that the Gauss curvature (2.6) vanishes everywhere but $\Gamma$ is not a geodesic on $\mathscr{L}$. In this section, we use the Hardy inequality of Theorem 3.1 to show that the presence of Gauss curvature prevents the spectrum to descend even if $\Gamma$ is mildly curved.

THEOREM 4.1. Given a positive number a and bounded continuous functions $\kappa, \tau$, and $\dot{\theta}$, let $\Omega$ be the Riemannian manifold (2.5) with the metric given by (2.3). Assume that $\tau-\dot{\theta}$ is not identically zero and that $a\|\tau-\dot{\theta}\|_{\infty}<\sqrt{2}$. Assume also that for all $s \in \mathbb{R}$,

$$
|\kappa(s) \cos \theta(s)| \leq \varepsilon(s):=\frac{\varepsilon_{0}}{1+s^{2}} \quad \text { with } \varepsilon_{0} \in\left[0, a^{-1}\right)
$$

Then there exists a positive number $C$ such that $\varepsilon_{0} \leq C$ implies that

$$
-\Delta_{D}^{\Omega} \geq E_{1}
$$

Here $C$ depends on $a$ and on the constants $c$ and $s_{0}$ of Theorem 3.1.

As usual, the inequality (4.2) is to be considered in the sense of forms. Actually, a stronger, Hardy-type inequality holds true, (cf. (4.7)).

An explicit lower bound for the constant $C$ is given by the estimates made in the proof of Theorem 4.1.

As a direct consequence of Theorem 4.1 , we get that the spectrum $\left[E_{1}, \infty\right)$ is stable as a set provided that the difference $\tau-\dot{\theta}$ vanishes at infinity.

Corollary 4.2. In addition to hypotheses of Theorem 4.1, assume that $\tau(s)-\dot{\theta}(s)$ tends to zero as $|s| \rightarrow \infty$. Then

$$
\operatorname{spec}\left(-\Delta_{D}^{\Omega}\right)=\left[E_{1}, \infty\right) .
$$

Proof. Following the proof of [4, Section 3.1] or [19, Section 5] based on a general characterization of essential spectrum adopted from [6], it is possible to show that the essential spectrum $-\Delta_{D}^{\Omega}$ coincides with the interval $\left[E_{1}, \infty\right)$, while Theorem 4.1 ensures that there is no spectrum below $E_{1}$.

Proof of Theorem 4.1. Let $\psi$ belong to $C_{0}^{\infty}(\mathbb{R} \times(-a, a))$. The proof is based on an algebraic comparison of $Q[\psi]-E_{1}\|\psi\|_{\mathscr{H}}^{2}$ with $Q_{0}[\psi]-E_{1}\|\psi\|_{\mathscr{H}}^{2}$ and the usage of Theorem 3.1. For 
every $(s, t) \in \mathbb{R} \times(-a, a)$, we have

$$
f_{-}(s):=\sqrt{1-\frac{a \varepsilon(s)[2+a \varepsilon(s)]}{1+a^{2}\|\tau-\dot{\theta}\|_{\infty}^{2}}} \leq \frac{h(s, t)}{h_{0}(s, t)} \leq \sqrt{1+a \varepsilon(s)[2+a \varepsilon(s)]}=: f_{+}(s) .
$$

Here the lower bound is well defined and positive provided that $\varepsilon_{0} \leq(3 a)^{-1}$, and both bounds behave as $1+O(\varepsilon(s))$ as $\varepsilon_{0} \rightarrow 0$; we keep the same notation $f_{ \pm}$for the functions $f_{ \pm} \otimes 1$ on $\mathbb{R} \times(-a, a)$. Consequently,

$$
\begin{aligned}
Q[\psi]-E_{1}\|\psi\|_{\mathscr{H}}^{2} \geq & \int_{\mathbb{R} \times(-a, a)} f_{+}^{-1} h_{0}^{-1}\left|\partial_{1} \psi\right|^{2} \\
& +\int_{\mathbb{R}} d s f_{-}(s) \int_{-a}^{a} d t h_{0}(s, t)\left(\left|\partial_{2} \psi(s, t)\right|^{2}-E_{1}|\psi(s, t)|^{2}\right) \\
& -E_{1} \int_{\mathbb{R} \times(-a, a)}\left(f_{+}-f_{-}\right) h_{0}|\psi|^{2} .
\end{aligned}
$$

Since the term in the second line is nonnegative due to (3.4) and Lemma 3.3, we can further estimate as follows:

$$
Q[\psi]-E_{1}\|\psi\|_{\mathscr{H}}^{2} \geq \min \left\{f_{+}(0)^{-1}, f_{-}(0)\right\}\left(Q_{0}[\psi]-E_{1}\|\psi\|_{\mathscr{H}_{0}}^{2}\right)-E_{1} \int_{\mathbb{R} \times(-a, a)}\left(f_{+}-f_{-}\right) h_{0}|\psi|^{2} .
$$

Using Theorem 3.1, we finally obtain

$$
Q[\psi]-E_{1}\|\psi\|_{\mathscr{H}}^{2} \geq\left\|w^{1 / 2} \psi\right\|_{\mathscr{H}_{0}}^{2},
$$

where

$$
w(s, t):=\frac{c \min \left\{f_{+}(0)^{-1}, f_{-}(0)\right\}}{1+\left(s-s_{0}\right)^{2}}-E_{1}\left[f_{+}(s)-f_{-}(s)\right]
$$

is positive for all sufficiently small $\varepsilon_{0}$.

\section{Acknowledgments}

This work has been supported by the Czech Academy of Sciences and its Grant Agency within the Projects IRP AV0Z10480505 and A100480501.

\section{References}

[1] D. Borisov, T. Ekholm, and H. Kovařík, Spectrum of the magnetic Schrödinger operator in a waveguide with combined boundary conditions, Annales Henri Poincaré 6 (2005), no. 2, 327-342.

[2] G. Carron, Inégalités de Hardy sur les variétés riemanniennes non-compactes, Journal de Mathématiques Pures et Appliquées. Neuvième Série 76 (1997), no. 10, 883-891.

[3] G. Carron, P. Exner, and D. Krejčiř́ik, Topologically nontrivial quantum layers, Journal of Mathematical Physics 45 (2004), no. 2, 774-784.

[4] B. Chenaud, P. Duclos, P. Freitas, and D. Krejčiř́ik, Geometrically induced discrete spectrum in curved tubes, Differential Geometry and Its Applications 23 (2005), no. 2, 95-105. 
[5] I. J. Clark and A. J. Bracken, Effective potentials of quantum strip waveguides and their dependence upon torsion, Journal of Physics. A 29 (1996), no. 2, 339-348.

[6] Y. Dermenjian, M. Durand, and V. Iftimie, Spectral analysis of an acoustic multistratified perturbed cylinder, Communications in Partial Differential Equations 23 (1998), no. 1-2, 141-169.

[7] J. Dittrich and J. Kříž, Curved planar quantum wires with Dirichlet and Neumann boundary conditions, Journal of Physics. A 35 (2002), no. 20, L269-L275.

[8] P. Duclos and P. Exner, Curvature-induced bound states in quantum waveguides in two and three dimensions, Reviews in Mathematical Physics 7 (1995), no. 1, 73-102.

[9] P. Duclos, P. Exner, and D. Krejčiř́ík, Bound states in curved quantum layers, Communications in Mathematical Physics 223 (2001), no. 1, 13-28.

[10] T. Ekholm and H. Kovařík, Stability of the magnetic Schrödinger operator in a waveguide, Communications in Partial Differential Equations 30 (2005), no. 4-6, 539-565.

[11] T. Ekholm, H. Kovařík, and D. Krejčiřík, A Hardy inequality in twisted waveguides, preprint, 2005, http://arxiv.org/abs/math-ph/0512050.

[12] M. Encinosa and L. Mott, Curvature-induced toroidal bound states, Physical Review A 68 (2003), 014102.

[13] P. Exner and P. Šeba, Bound states in curved quantum waveguides, Journal of Mathematical Physics 30 (1989), no. 11, 2574-2580.

[14] P. Freitas and D. Krejčirík, A lower bound to the spectral threshold in curved strips with Dirichlet and Robin boundary conditions, preprint, 2005.

[15] J. Goldstone and R. L. Jaffe, Bound states in twisting tubes, Physical Review B 45 (1992), no. 24, 14100-14107.

[16] D. Gridin, R. V. Craster, and A. T. I. Adamou, Trapped modes in curved elastic plates, Proceedings of the Royal Society of London. Series A. Mathematical, Physical and Engineering Sciences 461 (2005), no. 2056, 1181-1197.

[17] W. Klingenberg, A Course in Differential Geometry, Springer, New York, 1978.

[18] D. Krejčiŕík, Quantum strips on surfaces, Journal of Geometry and Physics 45 (2003), no. 1-2, 203-217.

[19] D. Krejčirík and J. Kř́z, On the spectrum of curved planar waveguides, Publications of Research Institute for Mathematical Sciences 41 (2005), no. 3, 757-791.

[20] Ch. Lin and Z. Lu, Existence of bound states for layers built over hypersurfaces in $\mathbb{R}^{n+1}$, preprint, 2004, http://arxiv.org/abs/math.DG/0402252.

[21] J. T. Londergan, J. P. Carini, and D. P. Murdock, Binding and Scattering in Two-Dimensional Systems, Lecture Notes in Physics, vol. m60, Springer, Berlin, 1999.

[22] B. Opic and A. Kufner, Hardy-Type Inequalities, Pitman Research Notes in Mathematics Series, vol. 219, Longman Scientific \& Technical, Harlow, 1990.

David Krejčiřík: Department of Theoretical Physics, Nuclear Physics Institute, Academy of Sciences of the Czech Republic, 25068 Řež, Czech Republic

E-mail address: krejcirik@ujf.cas.cz 